\title{
Severe Aplastic Anemia Presenting as Neutropenic Sepsis
}

\author{
Natasha Faye Daniels ${ }^{\mathrm{a}}$, Charlotte Burrin ${ }^{\mathrm{a}, \mathrm{c}}$, Raiiq Ridwan ${ }^{\mathrm{b}}$
}

\begin{abstract}
A 40-year-old female with aplastic anemia presented with a gum abscess deteriorating into neutropenic sepsis. Infection is an incredibly rare initial manifestation of aplastic anemia even on a background of significant neutropenia, hence the uniqueness of this case. The patient's initial complaints were of a subacute history of heavy vaginal bleeding and unexplained bruising, however on examination in the emergency department the patient was also noted to be pyrexial with gingival hyperplasia and a left sided submandibular lymphadenopathy. Initial blood results were phoned through from the lab reporting pancytopenia, confirming clinical suspicion of neutropenic sepsis. Antibiotic therapy was commenced and maxillofacial review for her unrelenting jaw pain revealed a gum abscess ultimately requiring tooth extraction. The patient underwent bone marrow biopsy showing hypocellular marrow with erythroid-dominant, dysplastic hematopoiesis. A thorough panel of investigations to rule out secondary causes led to the diagnosis of aplastic anemia, for which the patient is currently being managed with oral ciclosporin plus eltrombopagbridging therapy, plus counselling for the potential requirement for stem cell transplant.
\end{abstract}

Keywords: Aplastic anemia; Neutropenia; Sepsis; Pancytopenia; Atypical

\section{Introduction}

Aplastic anemia is a rare, diverse disorder characterized by pancytopenia and a hypocellular bone marrow in the absence of marrow fibrosis or infiltration. Diagnosis requires at least two of: hemoglobin $(\mathrm{Hb})<100 \mathrm{~g} / \mathrm{L}$; platelets $<50 \times 10^{9} / \mathrm{L}$; neutrophils $<1.5 \times 10^{9} / \mathrm{L}$ [1]. There are two peaks of the disease, at 15 - 25 years and again in over 60 years, and there

Manuscript submitted August 18, 2020, accepted August 25, 2020

Published online September 23, 2020

aFaculty of Medicine, University of Cambridge School of Clinical Medicine, Cambridge, UK

${ }^{b}$ Emergency Medicine, Cambridge University Hospitals NHS Foundation Trust, Cambridge, UK

${ }^{\mathrm{c} C o r r e s p o n d i n g ~ A u t h o r: ~ C h a r l o t t e ~ B u r r i n, ~ F a c u l t y ~ o f ~ M e d i c i n e, ~ U n i v e r s i t y ~}$ of Cambridge School of Clinical Medicine, 140 King's College, Cambridge, CB2 1ST, UK. Email: ceb211@cam.ac.uk

doi: https://doi.org/10.14740/jmc3570 appears to be a predisposition in individuals with histocompatibility locus human leukocyte antigen-DR isotype 2 (HLADR2) [2]. The vast majority of acquired cases are idiopathic, with no clear history of chemical, infectious or drug exposure prior to presentation. A significant minority of those where a trigger can be identified are found to have a preceding seronegative hepatitis [3]. The disease can present acutely or insidiously over months, most commonly with symptoms of anemia and thrombocytopenia. This case reports an extremely rare first presentation of aplastic anemia in the form of a neutropenic sepsis secondary to a gum abscess.

\section{Case Report}

A 40-year-old female presented to the emergency department with heavy per vaginal (PV) bleeding and dental pain. She reported significant PV bleeding with clots for the last 20 days prior to admission. Additionally, she had noted multiple unexplained bruises on her arms over the few weeks prior to admission. This was associated with shortness of breath on exertion, general malaise and a feeling of light-headedness. There was no relevant past medical history nor family history. On examination there were multiple areas of bruising with gingival hyperplasia and a left sided submandibular lymphadenopathy. Petechiae were seen on the roof of her palate and on her lips. She was subsequently found to be pyrexial $\left(38^{\circ} \mathrm{C}\right)$. Her abdomen was soft non-tender and there was no renal angle tenderness.

Initial bloods were taken, followed up by an urgent call from the laboratory reporting a pancytopenia with white blood cell $(\mathrm{WBC}) 1 \times 10^{9} / \mathrm{L}, \mathrm{Hb} 89 \mathrm{~g} / \mathrm{L}$, platelets $11 \times 10^{9} / \mathrm{L}$ and neutrophils $0.23 \times 10^{9} / \mathrm{L}$. A bone marrow biopsy was taken to investigate this further. This revealed hypocellular marrow with erythroid-dominant, dysplastic hematopoiesis. The suspicion of aplastic anemia was raised. Also seen was marked reduction in granulo- and megakaryopoiesis with no excess of blasts, as well as a degree of stromal edema. In addition, serum folate and vitamin B12 levels were notably reduced, giving a differential diagnosis of folate and B12 deficiencies. Both were supplemented, though this did not translate to any improvement in her blood count. A repeat bone marrow examination at this stage continued to show a hypocellular sample with erythroid-dominant hematopoiesis and markedly reduced granulopoiesis. Giemsa, Periodic acid-Schiff, Grocott's methenamine silver stain and Ziehl-Neelsen staining did not show any convincing evidence of intracellular parasites, fungal elements or acid-fast bacilli. Bone marrow deoxyribonucleic acid 
(DNA) samples performed found no DNA copy number abnormalities or loss of heterozygosity that could be interpreted as clinically significant. Screening for myeloma, antinuclear antibodies (ANA), anti-double stranded (ds) DNAs, anti-cyclic citrullinated peptide, rheumatoid factor, gamma-glutamyl transpeptidase, parvovirus immunoglobulin $\mathrm{G} / \mathrm{M}(\mathrm{IgG} / \mathrm{M})$ and polymerase chain reaction (PCR), beta-2-microglobulin, vitamin D deficiency, Igs, reticulocytes and glucose-6-phosphate dehydrogenase (G6PD) all came back negative. A diagnosis of idiopathic aplastic anemia was made.

Due to presence of neutropenia and fever, she was started on intravenous (IV) vancomycin and amikacin as per the hospital protocol for neutropenic sepsis for a patient with penicillin allergy. She subsequently developed increasingly severe pain in her left jaw which was not relieved by oral morphine. The next day she began spiking fevers (starting at $38.2^{\circ} \mathrm{C}$ and peaking at $38.9^{\circ} \mathrm{C}$ ) and complaining of ongoing pain, scoring it 9 out of 10. She was transfused with one pool of platelets which improved her platelets count to $32 \times 10^{9} / \mathrm{L}$. On day 3 she was again pyrexial with a temperature of $38.3^{\circ} \mathrm{C}$ at $5: 30 \mathrm{am}$. A maxillofacial review was performed given her dental pain, and LL8 gingival inflammation was noted with no bleeding or caries, prompting suspicion of a gum abscess. The patient remained febrile during the day and was passing clots with her urine. A further platelet transfusion was performed, however was complicated by rigors and tachycardia. Of six prior platelet transfusions, this was the first to cause a reaction and so it was decided that prior to her next platelet transfusion she would be premedicated with hydrocortisone and chlorphenamine.

She showed minimal response to a course of IV antibiotics and was once again febrile when IV antibiotics were stopped. A tooth extraction was therefore performed. Prolonged bleeding from the LL8 socket was reported despite a tranexamic acid-soaked gauze, followed by packing and suturing the socket. This eventually resolved with $1 \mathrm{~g}$ IV tranexamic acid and a further platelet infusion.

The patient was found to have HLA antibodies and thus was transfused with HLA-matched platelets to reduce the likelihood of further transfusion reactions. She clinically improved and was afebrile with no oromucosal bleeding or petechiae.

The patient was commenced on oral ciclosporin along with eltrombopag-bridging therapy in the hope that her blood count and marrow may improve on this therapy. She remains stable and is currently pending further definitive treatment such as $\mathrm{ATGAM}^{\circledR}$ or stem cell transplantation (SCT).

\section{Discussion}

Infection is an unusual presentation of aplastic anemia even with severe neutropenia, making this case very unique. Moreover, this patient presented with a very uncommon source of her septicaemia - a gum abscess. Patients with aplastic anemia are generally previously well, and the broad differential diagnosis for pancytopenia often requires bone marrow examination for definitive diagnosis. The definitive finding with such is an "empty" marrow: any marked hemophagocytosis, dysplasia or increased blasts would indicate alternative diagnoses, though differentiation from hypocellular myelodysplastic syndrome can be difficult. The disease course is variable, and treatment is based on the degree of cytopenia rather than marrow cellularity. In addition to supportive care with blood transfusions, prophylactic platelet transfusions and prophylactic antimicrobials, definitive management should be the aim with either immunosuppressive therapy or hematopoietic SCT (HSCT). First-line immunotherapy remains to be horse antithymocyte globulin (ATG) combined with ciclosporin. This provides hematologic recovery in $60-70 \%$ of cases with excellent long-term survival [4]. Administration of polyclonal antibodies such as ATG is complex and requires significant preparation and monitoring. HSCT is the alternative potentially curative treatment in severe disease or in cases where there is failure to respond to immunosuppressive therapy, though is not without significant risk. Age is a major determinant of success of this procedure, with risk increasing in patients above the age of 40 [5]. The source of stem cells also needs consideration, with graft-versus-host disease (GVHD) of greater incidence with grafts of peripheral blood origin than bone marrow, and unlike in the case of malignancy, where GVHD can offer graft-versus-tumour benefits, it must be avoided in aplastic anemia [6]. Outcomes with unrelated donor HSCTs are improving but are still inferior to those with matched sibling donors [7]. The major complications of aplastic anemia itself are due to neutropenia, leading to infection, and thrombocytopenia and resultant haemorrhage. Iatrogenic complications of both immunosuppressive therapy and bone marrow transplant must not be discounted.

Aplastic anemia is a disease with a very heterogeneous course. Some patients suffer only mild symptoms and do not require therapy. Rare cases such as this may present at the far end of the spectrum with neutropenic sepsis. The vast array of potentially unusual presentations, as in this case, require a low threshold for including aplastic anemia in one's differential diagnoses especially in cases of patients who present with unexplained bleeding and signs of infection. Failure to diagnose can lead to significant complications, and even death.

\section{Acknowledgments}

None to declare.

\section{Financial Disclosure}

None to declare.

\section{Conflict of Interest}

None to declare.

\section{Informed Consent}

Informed consent was provided by the patient. 


\section{Author Contributions}

Natasha Faye Daniels drafted, reviewed and revised the manuscript. Charlotte Burrin drafted, reviewed and revised the manuscript. Dr Ridwan reviewed and revised the manuscript.

\section{Data Availability}

The data supporting the findings of this study are available from the corresponding author upon reasonable request.

\section{References}

1. Guidelines for the diagnosis and management of adult aplastic anaemia. British Committee for Standards in Haematology. 2015. https://onlinelibrary.wiley.com/doi/ full/10.1111/bjh.13853.

2. Biswajit H, Pratim PP, Kumar ST, Shilpi S, Krishna GB, Aditi A. Aplastic anemia: a common hematological abnormality among peripheral pancytopenia. N Am J Med Sci. 2012;4(9):384-388.

3. Locasciulli A, Bacigalupo A, Bruno B, Montante B, Marsh J, Tichelli A, Socie G, et al. Hepatitis-associated aplastic anaemia: epidemiology and treatment results obtained in Europe. A report of The EBMT aplastic anaemia working party. Br J Haematol. 2010;149(6):890-895.

4. Rosenfeld SJ, Kimball J, Vining D, Young NS. Intensive immunosuppression with antithymocyte globulin and cyclosporine as treatment for severe acquired aplastic anemia. Blood. 1995;85(11):3058-3065.

5. Gupta V, Eapen M, Brazauskas R, Carreras J, Aljurf M, Gale RP, Hale GA, et al. Impact of age on outcomes after bone marrow transplantation for acquired aplastic anemia using HLA-matched sibling donors. Haematologica. 2010;95(12):2119-2125.

6. Chu R, Brazauskas R, Kan F, Bashey A, Bredeson C, Camitta B, Chiang KY, et al. Comparison of outcomes after transplantation of G-CSF-stimulated bone marrow grafts versus bone marrow or peripheral blood grafts from HLA-matched sibling donors for patients with severe aplastic anemia. Biol Blood Marrow Transplant. 2011;17(7):1018-1024.

7. Kim H, Kim BS, Kim DH, Hyun MS, Kim SH, Bae SH, Choi JH, et al. Comparison between matched related and alternative donors of allogeneic hematopoietic stem cells transplanted into adult patients with acquired aplastic anemia: multivariate and propensity score-matched analysis. Biol Blood Marrow Transplant. 2011;17(9):1289-1298. 